\title{
Beam Diagnostics Based on AC Modulation of System Parameters
}

\author{
Michael Tiefenback \\ Thomas Jefferson National Accelerator Facility, 12000 Jefferson Avenue, Newport News, VA \\ 23606, USA
}

\begin{abstract}
To improve the performance of operating accelerators, quantities such as lattice functions, beam transfer functions, betatron frequencies, etc, can be measured turn by turn with beam position monitors or from difference measurements using step changes in system parameters. Spectral measurements in closed orbit machines provide accurate values for some properties. But for open-ended systems and some measurements in closed-orbit machines, periodic modulation can be very useful for obtaining information about the beam line. Using examples from existing machines, we compare and contrast beam based modulation techniques and step function or passive measurements. For example, large amplitude dipole modulation in rings [1] can be used in dedicated exploration of nonlinear optical properties without beam degradation, even allowing for tune spread effects. Low-level modulation can provide real-time system monitoring with no adverse effect on beam users. Examples considered include fully resonant dipole modulation in storage rings such as RHIC (hadrons) and PEP-II (electrons), and the continuous low-level modulation used in the CEBAF recirculating electron linac for real-time feedback to improve availability.
\end{abstract}

\section{INTRODUCTION}

There are many ways to measure certain physical quantities. One can measure the height of a tree by cutting it down and using a tape measure from top to root. One can measure the current or charge in a beam by intercepting it on a suitably configured Faraday cup. Both of these measurements come at the price of destroying the item of interest. Noninvasive measurements often exist. The purpose of this paper is to discuss examples of measurements which involve AC modulation of beam or beam transport parameters, as opposed to simple step function changes, pointing out why the chosen technique is used and what tradeoffs are made in ease of use, avoiding degradation of beam, etc. This type of manipulation of beam or transport parameters is often called "dithering." The reasons for choosing such a system might be diverse: to provide a speedy update rate to facilitate initial tuning of system, to increase precision through averaging to allow for fine optimization, or to provide the ability to be used in a non-invasive manner to allow feedback compensation against drifts while users are making full use of the beam from the machine.

The mechanism is often to use the beam to sample properties of the beam transport system, diagnosing hardware behavior and configuration. However, the beam itself can be part of the transport system, with self- or beam-beam effects an intrinsic part of the beam environment. We include examples of both below. 
This technique opens many opportunities for improving measurements. By selecting a particularly "clean" portion (with respect to noise) of the frequency spectrum, and by using lock-in averaging techniques in signal analysis, one can enhance measurement precision by orders of magnitude. Or one can use the same enhancement of precision to reduce the perturbation amplitude until it becomes inconsequential to the users of the beam while still providing a useful monitor of system properties. One can sometimes use the same hardware to drive the beam at invasive levels, reducing the required signal averaging and resulting in essentially real-time updates for control room use, either for initial tuning or for troubleshooting.

\section{TYPICAL OUTLINE OF AC MODULATION MEASUREMENT}

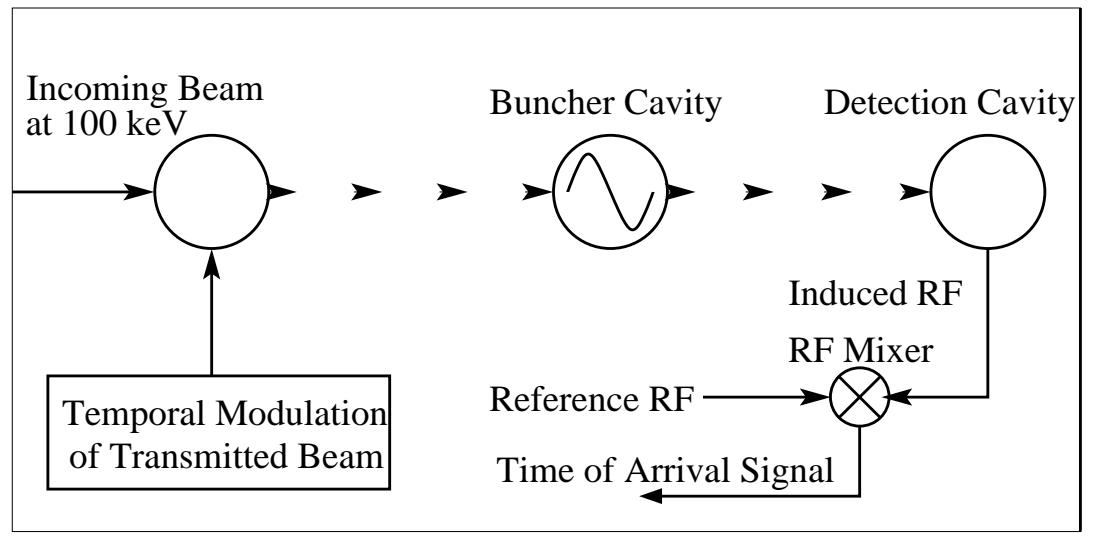

FIGURE 1. Schematic layout of modulation based diagnostic for a beam bunching region in a beam line. This system supports part of the tuning process for the CEBAF injector at Jefferson Lab. It is intrinsically destructive of the beam in that it relies upon ballistic propagation of longitudinal time-slices of the beam (longitudinal "rays") without the space-charge interactions present in the complete beam. See text for further discussion.

The basic layout of a modulation measurement is shown in Fig. 1 The figure shows a beam entering an RF deflection device, through which it is transmitted only for a selectable RF phase window[2]. The transmitted phase is modulated at acoustic frequencies. The induced RF in the detection cavity is mixed in quadrature against a master oscillator signal. The low-pass component of the mixed-down signal is proportional to variation in time of arrival of the beam particles. The bunching RF parameters are adjusted until the relationship between the beam time of arrival and the injection phase is as desired. Such a measurement can readily characterize the hardware configuration, aiding in-situ calibration and commissioning. However, this particular system is blind to longitudinal self-field effects within the beam. The measurement is made on longitudinal slices cut from the beam, each propagating in the absence of the others. 


\section{TYPICAL APPLICATIONS AND CONSIDERATIONS}

It is easy to incorporate AC modulated measurements when the quantities involved depend upon beam location or timing. Existing beam position monitors or relatively simple RF phase monitors can be used in conjunction with suitable reference sources for the parameter modulation. Off-the-shelf lock-in amplifiers or dedicated DSP hardware can be used to derive accurate correlation coefficients for the repetitive signals involved. Sometimes it is possible to adapt existing control and analysis hardware to do such tasks[3], taking better advantage of capabilities of the system.

Single- and multiple-particle oscillation frequencies, optical transfer functions, detection and localization of beam loss, relative timing between the beam and RF fields, calibration of those RF fields, and differential energy gain (vs. RF phase) are ready candidates for measurement. As is often the case, tuning to null points in response can provide a very precise reference for calibration of hardware either for periodic checks or for recalibration after maintenance or other replacement of components.

As with any system, the relative capital cost of installation is one of the factors determining what actually gets installed. But other issues must also be included in the discussion. Everything costs money, and some tradeoffs are subtle and affect the installation for the long haul. Ease of use and ease of interpretation of diagnostics are worth considering. Difficult-to-use diagnostics are not used as often as easy ones. Signals which require care in interpretation are often mis-interpreted and cause unnecessary down time for the accelerator installation. The performance of modulation-based diagnostics can be adjusted within certain limits through control of the perturbation amplitude and of the integration time used to filter noise. The effective update rate in the control room can be enhanced for manual tuning or troubleshooting activities.

Another consideration is degradation of the beam. For open-ended systems, like linacs, beam degradation during setup or troubleshooting exercises may be acceptable, particularly at reduced duty factor. However, for hadron storage rings (such as RHIC), degradation of the beam emittance is not reversible and necessitates refilling the ring. Some optics measurements which are difficult to do even in this case are enabled by RF dipole techniques $[1,4]$.

A diagnostic device which requires dedicated beam time consumes time which could otherwise be dedicated to user experiments. One system discussed below for which this is clearly an area of benefit is the on-line transit time adjustment system at Jefferson Lab [5]. Beam time of roughly $1 \mathrm{hr}$ per week was returned to the users after this system became fully operational, replacing an invasive tuning procedure.

Table 1 outlines some techniques of measuring transverse beam transfer functions and related parameters in rings and linacs. The differences in behavior between open- and closed-orbit machines affect the techniques of choice. Linac transfer functions can be easy to measure when the beam is not required to be simultaneously delivered to users. When it is advantageous for measurements to be made during routine beam delivery, small perturbations and feedback at the target or interaction region are very helpful. Use of correlation/lock-in techniques with low-level modulation of the beam steering can give highly accurate values for transfer functions when, for instance, some aspect of the transfer function is required to remain fixed. Closed-orbit systems require extra care as the perturbation does not convect out of the machine as it does for open systems. 
TABLE 1. Miscellaneous transfer function measurements in rings and linacs

\begin{tabular}{|c|c|c|}
\hline $\begin{array}{l}\text { Non-repetitive: } \\
\text { Standard Technique }\end{array}$ & Ring & Linac \\
\hline $\begin{array}{l}\text { Impulse kick: } \\
\text { transfer functions } \\
\text { via difference orbit }\end{array}$ & $\begin{array}{l}\text { Turn by turn bpm } \\
\text { measurements } \\
\text { Disturbance disperses } \\
\text { with time }\end{array}$ & $\begin{array}{l}\text { Transient bpm } \\
\text { measurements } \\
\text { Disturbance convects } \\
\text { out of system }\end{array}$ \\
\hline $\begin{array}{l}\text { Static corrector kick: } \\
\text { transfer functions via } \\
\text { difference orbits }\end{array}$ & $\begin{array}{l}\text { Kick can affect global system: } \\
\text { e.g., must compensate any } \\
\text { change in circumference }\end{array}$ & $\begin{array}{l}\text { Difference orbits can } \\
\text { be made large and precisely } \\
\text { measured from bpm averages }\end{array}$ \\
\hline \multicolumn{3}{|l|}{ AC-Modulated } \\
\hline $\begin{array}{l}\text { Phase locked loop } \\
\text { excitation/detection }\end{array}$ & $\begin{array}{l}\text { Low amplitude modulation } \\
\text { locked onto betatron resonances } \\
{[6,7,8]}\end{array}$ & $\begin{array}{l}\text { No direct linac analog } \\
\text { (no betatron resonance) } \\
\text { Perhaps sub-threshold Beam } \\
\text { Break-Up gain measurement? }\end{array}$ \\
\hline AC Dipole & $\begin{array}{l}\text { Special treatment for rings } \\
\text { Kick near betatron resonance } \\
\text { in ring can provide stable } \\
\text { harmonic orbit excursion } \\
\text { Synchronous detection } \\
\text { may make nonlinear ring } \\
\text { dynamics accessible } \\
{[1,4]}\end{array}$ & 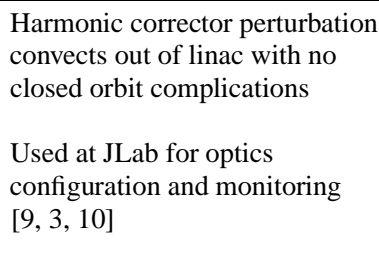 \\
\hline
\end{tabular}

\section{BETATRON TUNE MONITORING IN RINGS}

Because of the central importance of resonances in rings, the betatron tune is a critical parameter. Tune-shift effects from various sources can drive the beam to cross resonances and degrade the beam, decreasing the performance of storage rings. Measurement of the betatron tune in rings has been improved in recent years by application of lock-in techniques involving driving the beam directly on the resonance and measuring essentially an $S_{21}$ transfer function $[6,7,8]$. Locking to the frequency for peak $S_{21}$, RHIC with its hadron beam and no significant synchrotron light damping, and PEP-II with its high-current, feedback stabilized beam of electrons with heavy synchrotron light damping are both able to make similar measurements.

This beam-based measurement perturbs the beam particles without otherwise perturbing the environment of the beam. The effects of space-charge, wall and wake-field interactions, beam-beam tune shift, and so forth are all involved in the measured betatron tune. As schematically illustrated in Fig. 2, an RF transverse kick is applied to the beam somewhere in the ring and a pick-up elsewhere in the machine senses the spectral component of the beam motion at the kick frequency. Narrow band, off-the-shelf lockin hardware and special-purpose signal processors all have their place in such systems. Lower noise levels in the pick-up circuit and increased sensitivity in the detector both contribute to allowing for reductions in the perturbing kicker signal, and restricting the spectral breadth of the perturbation also contributes to reducing the total perturbation energy available to degrade the beam. The PEP-II example was chosen to illustrate its 


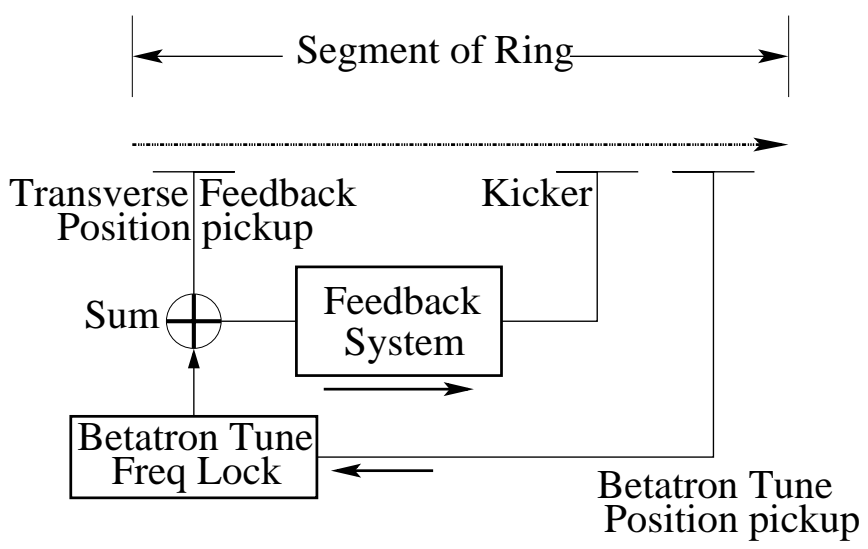

FIGURE 2. The PEP-II betatron tune system, schematically illustrated. The transverse feedback system is used to drive the betatron frequency perturbation on the beam, as it already exists and can be incorporated without degrading its own performance.

re-use of the existing transverse stabilization feedback kicker for diagnostic purposes, simultaneously with its original use.

\section{SYSTEM SETUP EXAMPLES AT JEFFERSON LAB}

In addition to the example in Fig. 1, other setup tools at Jefferson Lab are modulationbased. The setup tools are used periodically and their operation is generally inconsistent with simultaneous beam delivery. The buncher setup system is used to gather a stream of correlation data between the injection timing and time of arrival at the downstream detector. An example correlation plot is shown in Fig. 3. The plot shows a roughly 1 picosecond variation in arrival time on the horizontal axis associated with a roughly 110 picosecond variation in injection time on the vertical axis. The phase compression measured here is not a direct measure of the bunch length, but of the configuration of the bunching RF system parameters and the transmission window set to center on the longitudinal acceptance of the buncher. Particles arriving either too early or too late fall outside of the acceptance and end up as late arrivals at the detection cavity. This presentation gives operators an idea of what the system is doing. Sampling a wide range of injection phase and showing the results for the slices provides a picture of the performance of the system much better than any single scalar measurement.

A similar time of arrival detection system is in place at the end of each of the two CEBAF linacs. During pulsed beam setup work, part of the pulse structure is a short post-pulse of duration equal to the electron transit time around the accelerator, about four microseconds. The detection cavities are installed on the common portion of the beam line occupied by the beam on each circuit of the accelerator. The signal from each of the cavities provides a relative time-of-flight signal for the successive passes of the 


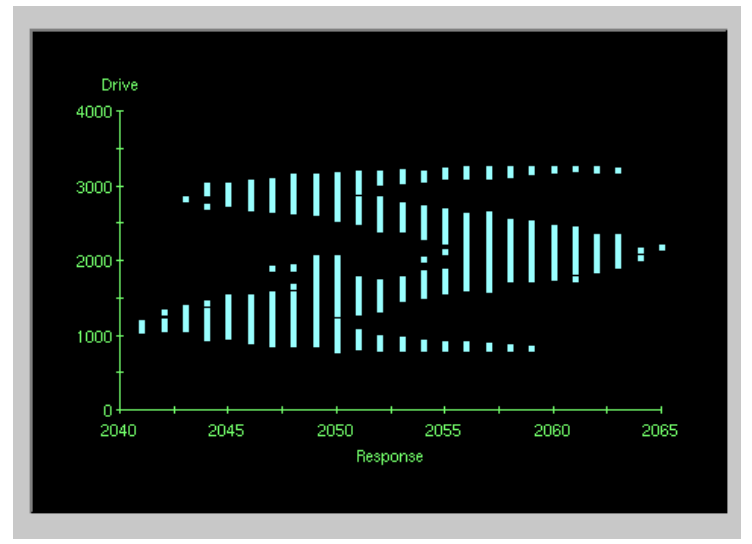

FIGURE 3. Correlation plot of time of arrival (horizontal axis; full scale approximately 1 picosecond) vs. time of injection (vertical axis; full extent of data approximately 110 picoseconds). The residual distortions from a straight line are because of aberrations in the longitudinal focus and some intentional over-focusing.

beam. This static setup tool is also used with a modulation of beam energy to detect excessive time-of-flight variations ( $R_{56}$ or $M_{56}$ transfer matrix element).

Tuning of the optics of the machine is the largest single activity in machine setup at Jefferson Lab. Because the machine is essentially a folded linac, open-ended, resonance effects are much milder than the betatron resonance issues in closed-orbit machines. Phase advance requirements on transfer functions are very relaxed as a result. Control of the peak values of the envelope functions is very important, however. At defined locations, correctors are excited with $30 \mathrm{~Hz}$ signals locked to the $60 \mathrm{~Hz}$ frequency of the AC power grid. The resulting $30 \mathrm{~Hz}$ differential orbit variations are detected by the beam position monitor (BPM) system.

These synchronous position variations are fitted in groups of from 3 to $5 \mathrm{bpms}$ to determine $\left(\mathrm{x}, \mathrm{x}^{\prime}, \mathrm{y}, \mathrm{y}^{\prime}\right)$ values for the beam centroid along the machine relative to the unperturbed beam [9]. The actual number of oscillations executed by a particle on its path is not of great importance. What is important is control of the Twiss functions $\beta$ and $\alpha$ along the accelerator. From the differential orbit data and beam momentum, one derives the emittance-like constant of the motion for each of the two linearly independent orbit perturbations in each plane. An example of the resulting plots is shown in Fig. 4. The vertical scale is arbitrary, and the horizontal dimension is simply distance of propagation through the accelerator. The yellow lines should, in the ideal case, all be featureless horizontal lines. Deviations from ideal are tuned back toward the desired value using quadrupoles defined for this purpose in the optics. This tuning is done with millimeter scale orbit perturbations, and in pulsed beam mode so that accidental scrapage of beam will not damage hardware. The update interval of the diagnostic as presently implemented is long: about 15-20 seconds to fully cycle through all four orbit perturbations. 


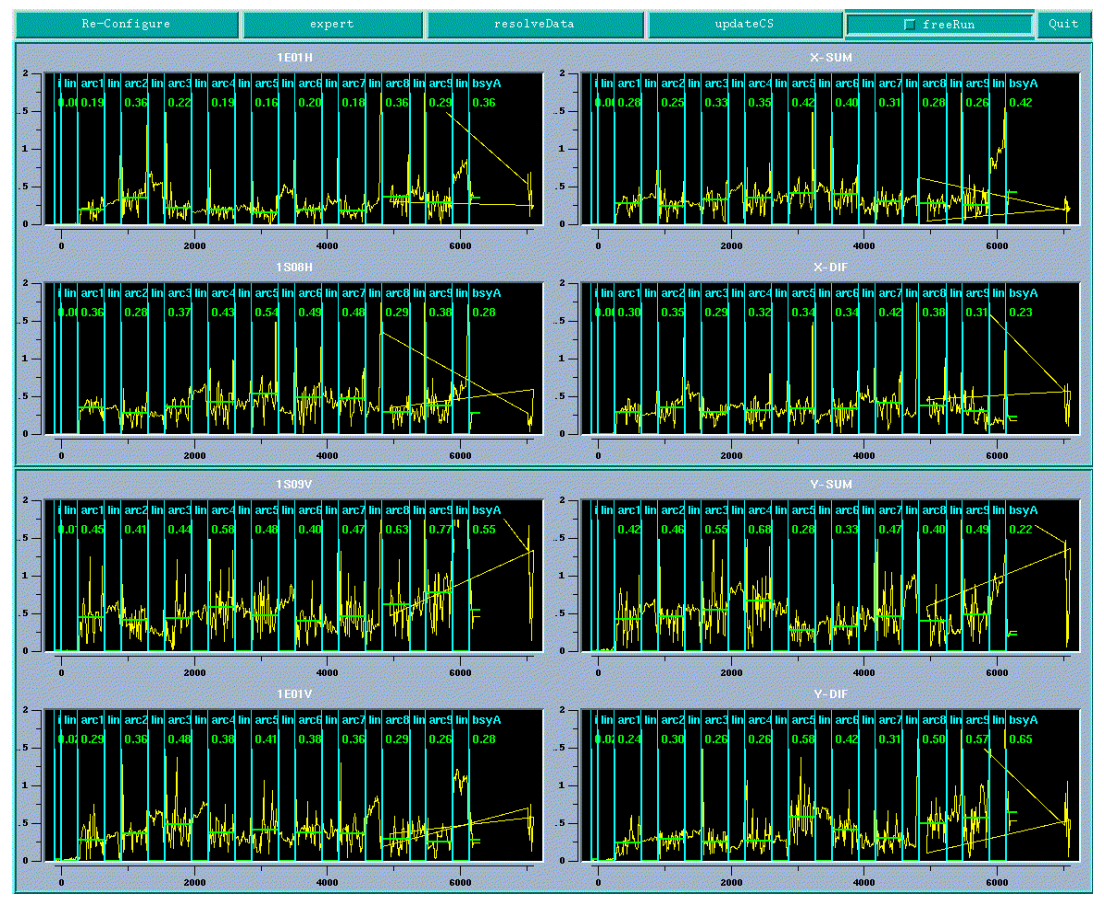

FIGURE 4. The emittance-like Courant-Snyder constant for each of four perturbed orbits through the CEBAF accelerator. The left-hand column shows the results for independent orbits, while the right-hand column shows numerically combined sums and differences for the respective planes. The vertical scale is arbitrary, and the horizontal scale is distance of propagation through the accelerator. In the ideal case, the yellow lines would be flat for each orbit.

\section{ON-LINE EXAMPLES AT JEFFERSON LAB}

\section{Beam Scraping Monitor}

In addition to the setup mode diagnostics, there are several related systems which are designed for constant monitoring of the accelerator during beam delivery. The Beam Scraping Monitor [10] uses low-level modulation of beam position (tens of microns) to search for chronic beam scraping on an obstacle. This could occur from an insertable beam line element failing to retract properly or from drifts in beam position allowing the beam to be too close to a material object like a septum. The absolute sensitivity of the beam current detectors is at the 100 nanoampere level, but drifts of that level also occasionally are found. The incremental sensitivity of the beam current detectors should be quite reliable. We have supplemented the $\mathrm{CW}$ beam loss protection system, which requires the total current to all users to match the current injected into the linacs, with a fundamentally different system which searches for beam current modulation at any Hall that is synchronous with the applied steering perturbation(s). A cartoon of the principle 
is shown in Fig. 5. The beam is traveling to the right. The portion of the beam that passed the obstruction earlier, which it was deflected toward the obstruction, lost some of its charge on the obstruction. The modulation in beam current is synchronous with the steering modulation.

\section{Loss is Synchronous with Transverse Modulation}

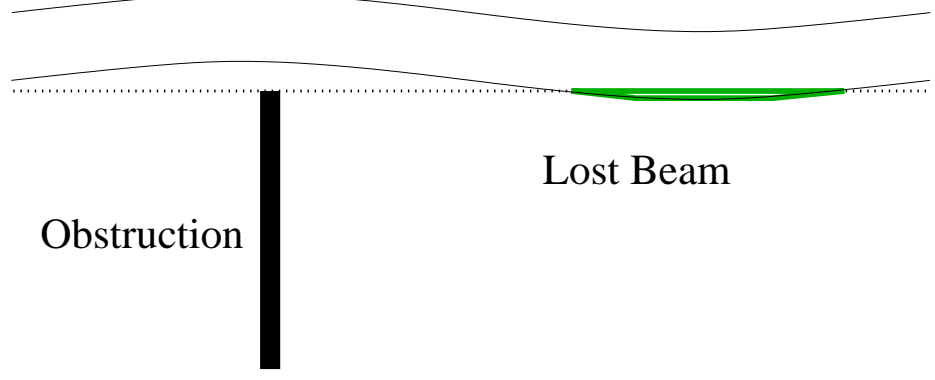

FIGURE 5. The Beam Scraping Monitor: the beam position is modulated at the scale of tens of microns. Should the beam be near an obstacle which is intercepting a significant portion of its current, an AC signal is generated in the beam current monitor hardware. A sufficiently large beam loss correlated with the steering perturbations raises a warning to the operations crew that a portion of the beam is probably being scraped by an obstruction.

Four independent modulation sources are used in this system, each at its own unique frequency for easy determination of which corrector perturbation is driving the beam loss. In this instance, the detector need only have an accurate independent time base; only the frequency is used for correlation. The relative phase of the beam loss and the perturbation are systematically ignored. This choice meant that it was not necessary to provide an analog connection to link the reference signal to the lock-in amplifier.

\section{Transfer Function Monitor}

The BSM monitors the consequences of its perturbations of the beam on the transmitted beam current. These perturbations also result in beam position correlations in the final beam transport lines to the experiments. Monitoring of the differential orbits induced by the BSM provides a measure of the overall transverse transfer function between the correctors and the final bpms. Although the Fast FeedBack (FFB) system [11] by design has negligible gain at the frequencies chosen for the BSM $(600-800 \mathrm{~Hz})$, it does provide a good monitoring and correlation tool. The purpose of this monitor is to provide early warning of changes in the optics along the accelerator, notifying operations when and whether a change has occurred, rather than where the change occurred. Such a monitor is useful because no other accelerator diagnostic can diagnose distributed changes in the machine optics such as are caused by transient faults in the power supplies and occasionally in the electrical utility grid. It is also a test application for the idea of distribution correlation capability generically in portions of the BPM system. If the 
Four independent harmonic correctors

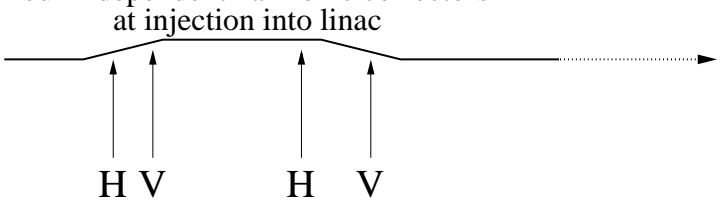

different frequencies for separation

Multiple synchronous BPMs separate frequencies

Fit each resulting orbit to local optics in transfer line

Obtain transfer function from injector to experiment

Uses existing BSM modulations

FIGURE 6. The Transfer Function Monitor: the same perturbations imposed by the Beam Scraping Monitor are detected in the experimental lines by the FFB system.

BPM system were able to extract differential orbit data accurately during beam delivery for experiments, the optics of the machine could be monitored for early detection of transient magnet power supply faults or other difficult to find faults.

\section{Global RF Phase and Transit Time Correction}

In a phase-stable accelerator, the RF systems provide a timing reference to which the beam is dynamically self-locked. The CEBAF electron linac uses on-crest acceleration with a short injected bunch to minimize energy spread for the users. The electrical master oscillator distribution system was found empirically to be insufficiently stable over the span of minutes to guarantee the required energy spread and energy stability. The Master Oscillater Modulator (MOMod) system [12] was installed to allow measurement and correction of the relative timing of beam and RF in each linac as a whole, not requiring phase correction of each cavity individually.

A small $\left( \pm 0.05^{\circ}\right.$ peak-peak $)$ phase modulation, considerably smaller than the typical full bunch length of $0.5^{\circ}$, induces a small energy modulation on the beam, sufficient to be detectable using lock-in amplifier techniques but otherwise inconsequential. This system is shown schematically in Fig. 7. The phase difference $\psi$ between the beam and the RF and the energy modulation $\delta E$ synchronous with the phase modulation are related through

$$
\delta E \propto \sin (\psi) .
$$

The lock-in amplifier can monitor not only the magnitude of the energy modulation but also its sign relative to the RF phase modulation. Regulation of phase errors to $\pm 0.12^{\circ}$ is readily achieved, as shown in Fig. 8, tracking through seasonal drifts of tens of degrees. Typical short-term drifts in the absence of regulation are at the $\pm 1^{\circ}$ level.

In addition, ground motion through the calendar year, as well as thermal expansion (particularly after extended maintenance periods), caused variability in the pass-to-pass 


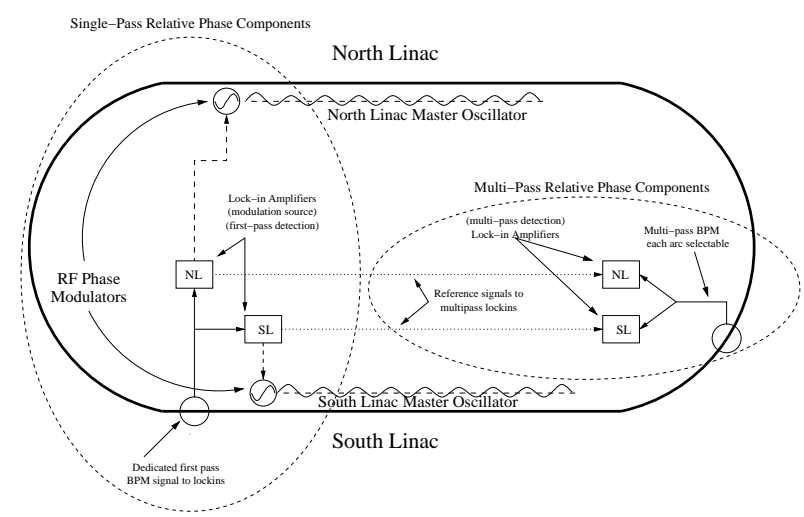

FIGURE 7. The overall layout of beam to RF timing system (MOMod) used to maintain the RF synchronous with the first pass beam and the higher pass system used to monitor variations in the transit time of the beam around the accelerator.

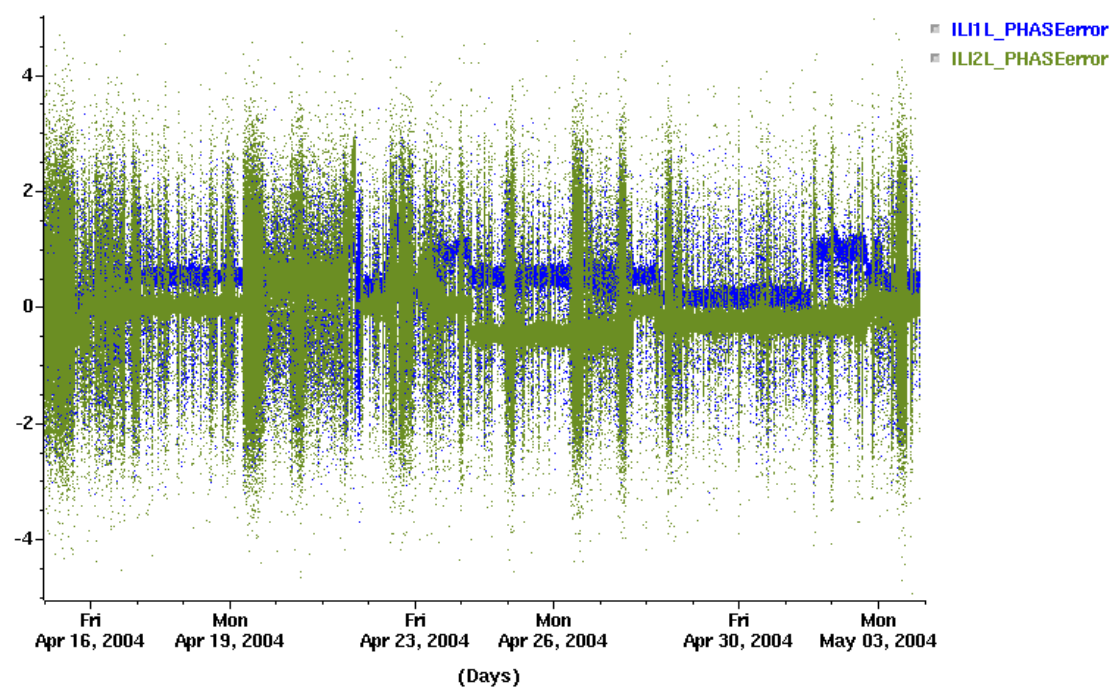

FIGURE 8. Display of regulated linac phase error. Large indicated errors are a combination of real errors measured after beam restoration and before regulation is enabled, as well as noise during the absence of beam. The discrete steps in the measured phase were due to manual setpoint changes made in attempts to trade off RF phase against transit time errors and maintain energy spread. The vertical scale is degrees of phase at $1497 \mathrm{MHz}$.

transit time. The setup tools used in the early running of the accelerator were not usable during experimental beam delivery, and they required more beam time for correction of these drifts than was desirable. A non-invasive monitor for beam transit time was devised using the same RF phase modulation applied to monitor the first-pass phase relationship 


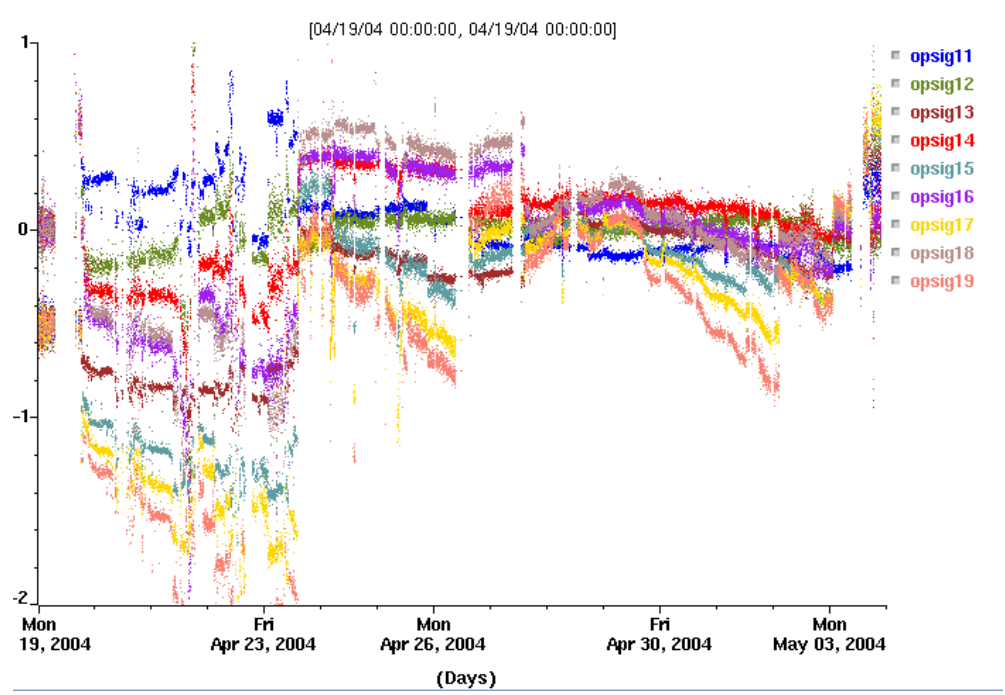

FIGURE 9. Display of the relative phase of the beam on various recirculation passes during a two-week interval, showing several discrete correction events. The vertical scale is again degrees of phase at 1497 $\mathrm{MHz}$. The accelerator is expanding, so the beam arrives after the RF peak.

between the RF and beam. This phase modulation continues to affect the energy of the beam through the remainder of its acceleration. With additional pickup locations (at dispersive points) on the higher passes, it is possible to measure transit time slippage of the beam on subsequent recirculations. Such slippage is the result of change in effective machine circumference. Real-time, non-invasive measurement allows operations staff to compensate for changes in the machine circumference using the chicanes placed along the machine. Examples of this are shown in Fig. 9. These systems are part of the supporting diagnostics which are required to deliver reliably a relative energy spread under $2 \times 10^{-5}$.

\section{UPGRADES AND POTENTIAL ADDITIONS}

\section{CEBAF Individual Cavity Phases}

The field of each of the individual cavities should be at peak when the beam transits the cavity. The cavity cresting protocol which had been in routine use in the CEBAF accelerator induced too much transient energy deviation to satisfy the users as their requirements became more stringent. An upgraded AC-modulated system can eliminate this interference and provide improved performance at the same time. The former protocol [13] used discrete changes in cavity phase and measured the induced beam energy shift to determine optimum phase. By using a sinusoidal modulation instead of stepfunction changes, high frequency noise in the system is reduced and the FFB system can 
compensate for the variation in beam energy at the experimental stations. Ten $\mathrm{Hz}$ would be a convenient frequency for this phase modulation. The FFB error signal provides the data from which the beam phase error would be derived. This involves essentially the same algorithm as is used for setting each linac global RF phase, but implementing the phase modulation on a per-cavity basis. At the infrequent times when time is taken to correct the individual cavity phases, the linac commonly increases its energy gain by $1 / 2 \%$ and the overall gradients in the cavities must be reduced by this amount. Of somewhat greater concern are the occasional large phase errors found in such activities, which would be found much more rapidly by routine non-invasive monitoring.

\section{Proton Linac Time of Flight Example}

In an electron linac, dispersive regions are the obvious choice for detecting momentum changes of the beam. In mildly relativistic conditions, the same pattern of cavity phase modulation mentioned in the CEBAF upgrade scenario can be coupled with a time of arrival monitor of the kind used in the bunch compression example. This would provide a time of flight monitor which could be used to determine the phase of RF cavities. The example we will consider is patterned after the Spallation Neutron Source (SNS) parameters.

The roughly 100 femtosecond resolution of CEBAF cavities at 10 microamperes of beam current should be improved at higher beam current. Presuming a drift of roughly 15 meters from the acceleration cavity to a sensing cavity downstream, an energy gain from each cavity between $6 \mathrm{MeV}$ and $10 \mathrm{MeV}$, and allowing up to $\pm 5^{\circ}$ of phase modulation, one should readily be able to measure phase errors of $1^{\circ}$ from crest phase. As such linacs generally operate well away from crest phase in phase stable operation, and the signal is proportional to the sine of the off-crest phase, the sensitivity will be large. This system would target a specific value of the differential time of flight instead of the null point at peak energy gain. Commissioning experience at CEBAF indicates that use of a detection system with little or no positional dependence is wise, as beam steering effects at low beam energy associated with the cavity phase modulation were difficult to separate from real time of flight variations. For a CW machine with short bunches, the Fourier amplitudes of the RF harmonics are essentially as high as that of the fundamental frequency. Using overmoded pickup cavities can improve the timing sensitivity. The 6th harmonic $6 \mathrm{GHz}$ cavity used in some applications at Jefferson Lab is very close to the 8th harmonic of many of the SNS cavities (805 MHz), for instance.

\section{CONCLUSION}

AC modulation techniques can provide readily interpretable pictures of system performance for use in the control room. The same system hardware can often be configurable for setup purposes to speed update rates or for high precision, lower bandwidth measurements for accurate feedback control during user delivery. 


\section{REFERENCES}

1. Peggs, S., and Tang, C., Nonlinear Diagnostics using an AC Dipole, Tech. Rep. RHIC-AP-159, Brookhaven National Laboratory, Upton, NY, USA (1998).

2. Abbott, R. et al., "The CEBAF Injector RF Distribution and Bunch Length Measurement System," in Proceedings of the 1992 International Linac Conference, AECL-10728, 24-28 Aug, 1992, edited by C. R. Hoffmann, AECL, Chalk River, Ontario, Canada, 1992, p. 232.

3. Dickson, R., "Development of a BPM Lock-In Diagnostic System," in Proceedings of the 2003 Particle Accelerator Conference, 12-16 May, 2003, Portland, Oregon, USA, edited by J. Chew, P. Lucas, and S. Webber, IEEE, 2003, pp. 2563-2564.

4. Bai, M. et al., "Measuring Beta Function and Phase Advance in RHIC with an AC Dipole," in Proceedings of the 2003 Particle Accelerator Conference, 12-16 May, 2003, Portland, Oregon, USA, edited by J. Chew, P. Lucas, and S. Webber, IEEE, 2003, pp. 2204-2206.

5. Tiefenback, M., "On-Line Measurement and Tuning of Multi-Pass Recirculation Time in the CEBAF Linacs," in Proceedings of the 2001 Particle Accelerator Conference, 18-22 June, 2001, Chicago, Illinois, USA, edited by P. Lucas and S. Webber, IEEE, 2001, pp. 553-555.

6. Brennan, M. et al., "Tune Measurement in RHIC," in Beam Instrumentation Workshop 2002: Tenth Workshop, edited by G. A. Smith and T. Russo, AIP Conference Proceedings 648, American Institute of Physics, New York, 2002, p. 134.

7. Cameron, P. et al., "RHIC Third Generation PLL Tune System," in Proceedings of the 2003 Particle Accelerator Conference, 12-16 May, 2003, Portland, Oregon, USA, edited by J. Chew, P. Lucas, and S. Webber, IEEE, 2003, pp. 524-526.

8. Fisher, A. S. et al., "Upgrades to PEP-II Tune Measurements," in Beam Instrumentation Workshop 2002: Tenth Workshop, edited by G. A. Smith and T. Russo, AIP Conference Proceedings 648, American Institute of Physics, New York, 2002, p. 267.

9. Lebedev, V. A. et al., "Linear Optics Correction in the CEBAF Accelerator," in Proceedings of the 1997 Particle Accelerator Conference, 12-16 May, 1999, Vancouver, B. C., Canada, edited by M. C. et al., IEEE, 1997, pp. 2128-2130.

10. Allison, T. et al., "The CEBAF Beam Scraping Monitor," in Proceedings of the 2001 Particle Accelerator Conference, 18-22 June, 2001, Chicago, Illinois, USA, edited by S. W. P. Lucas, IEEE, 2001, pp. 2398-2391.

11. Dickson, R. A., and Lebedev, V. A., "Fast Digital Feedback System for Energy and Beam Position Stabilization," in Proceedings of the 1999 Particle Accelerator Conference, 27 March - 2 April, 1999, Chicago, Illinois, USA, edited by A. Luccio and W. MacKay, IEEE, 1999, pp. 646-648.

12. Lebedev, V. et al., "High-Precision Beam-Based RF Phase Stabilization at Jefferson Lab," in Proceedings of the 1999 Particle Accelerator Conference, 27 March - 2 April, 1999, Chicago, Illinois, USA, edited by W. M. A. Luccio, IEEE, 1999, pp. 1183-1185.

13. Tiefenback, M. G., and Brown, K., "Beam-Based Phase Monitoring and Gradient Calibration of Jefferson Laboratory RF Systems," in Proceedings of the 1997 Particle Accelerator Conference, 12-16 May, 1999, Vancouver, B. C., Canada, edited by M. C. et al., IEEE, 1997, pp. 2271-2273. 